\title{
Imaging diagnosis for bisphosphonate-related osteonecrosis of the jaw (BRONJ): are there any prospective findings or not?
}

(C) Japanese Society for Oral and Maxillofacial Radiology and Springer Japan 2013

At the 17th Congress of Clinical Imaging for Oral and Maxillofacial Lesions of the Japanese Society for Oral and Maxillofacial Radiology, Professor Kimishige Shimizutani, the president, held a symposium entitled "Imaging diagnosis for bisphosphonate-related osteonecrosis of the jaw (BRONJ): are there any prospective findings or not?"

Akira Taguchi coordinated the symposium. Satoru Toyosawa, an oral pathologist, introduced the histopathology of BRONJ. Yoshikazu Suei analyzed the radiographic findings of BRONJ. Yoshiko Ariji analyzed the magnetic resonance images of BRONJ. Makoto Tsuchimochi analyzed the nuclear medicine images of BRONJ.

The editor felt that this symposium was very important and has decided to publish its contents in Oral Radiology. He believes that this topic will be attractive for all readers of the journal.

Keiji Tanimoto, Editor-in-Chief 